\title{
An Optimization Model for Operations of Large-scale Hydro Power Plants
}

\author{
Gonzalo E. Alvarez
}

\begin{abstract}
Globally, there is an increase in the proportion of renewable sources for electricity generation. Among renewable sources, hydropower is the most widespread. For this reason, the improvements of their applications have been the focus of researches. Hydroelectric power plants have numerous aspects which might represent several economic advantages, if they are operated efficiently. Mathematical optimization models are interesting tools that help in the decision-making processes. In this context, this paper introduces a new Mixed Integer Lineal Programming model that determines the most convenient combination of units to operate a large-scale hydro power plant. Several aspects of reality are taken into account, which are sometimes not considered, such as the variation of the hydraulic head and the performance of other elements besides the turbines, as floodgates. To prove the effectiveness of the new model, the Itaipú Power Plant is selected as a case study. It has an installed power capacity of 14,000 MW and holds the world record in terms of annual generation with 103 million MWh. Three possible scenarios are evaluated in order to analyze the behavior of this plant in normal and extreme situations. The results indicate that the model effectively reduces computational times, and that power generation is influenced by market price variations and reservoir limitations.
\end{abstract}

Index Terms-Mixed Integer Linear Programming, Itaipú, Hydraulic Head, Operating Curves, Electric Power Generation

\section{NOMENCLATURA}

Índices:

$i \quad$ Unidad generadora

$t \quad$ Unidad de tiempo

ss $\quad$ Nivel de salto hidráulico

gs Segmento de generación

C

Constantes:

$\rho_{i}^{p p} \quad$ Precio horario de generación (USD/MW)

$\sigma_{i} \quad$ Costo de la potencia unitaria producida (USD/MW)

$c_{i}^{S U} / c_{i}^{S D} \quad$ Costo de encendido de la unidad (USD)

$D_{t} \quad$ Demanda programada (MW)

$r f_{\text {in }} / r f_{\text {out }} \quad$ Caudal entrante/saliente del río $\left(\mathrm{Hm}^{3} / \mathrm{h}\right)$

$v_{s S}^{\min } / v_{s S}^{\max }$ Nivel mínimo/máximo de volumen que pertenece al salto ss $\left(\mathrm{Hm}^{3}\right)$

Gonzalo E. Alvarez, INGAR/CONICET-UTN, Instituto de Desarrollo y Diseño, Avellaneda 3657, Santa Fe, Argentina, (e-mail: galvarez@santafeconicet.gov.ar).

$S_{s s}^{\max } / S_{s s}^{\min }$
$\Delta p_{s s, g s, i}$
$\Delta \delta_{s s, g s, i}$
$\overline{p_{\imath} / p_{i}}$
$\operatorname{Variables:}$
$\rho_{i}^{p p}$
$S U_{i, t} / S D_{i, t}$
$\delta_{i, t}$
$S_{i, t}$
$e_{t}$
$Z_{i, t}$
$\phi_{s s, g s, i, t}$
$S H_{s s, t}$
$\delta v_{c, t}$

Máximo/mínimo valor de salto (m) Valor de potencia generada de muestra (MW) Valor de descarga del punto de muestra $\left(\mathrm{Hm}^{3} / \mathrm{h}\right)$ Limites operativos (MW)

Potencia generada (MW)

Binaria que vale 1 cuando la unidad se enciende/apaga

Descarga de la unidad $i\left(\mathrm{Hm}^{3} / \mathrm{h}\right)$

Salto hidráulico de la unidad $i(\mathrm{~m})$

Volumen del embalse $\left(\mathrm{Hm}^{3}\right)$

Binaria de estado de la unidad $i$

Variable de peso de generación $[0,1]$

Binaria para la pertenencia al nivel de salto

Descarga de la compuerta $\left(\mathrm{Hm}^{3} / \mathrm{h}\right)$

\section{INTRODUCCIÓN}

a generación eléctrica a partir de fuentes renovables ha crecido en proporción al total mundial de las fuentes en los últimos 40 años [1]. La fuente hidráulica es la que tiene mayor predominancia de las energías renovables utilizadas para la generación de electricidad, llegando a representar el $16,6 \%$ de la generación mundial en 2015 y el $70 \%$ de la generación renovable [2]. En la actualidad, existen centrales hidroeléctricas en más de 150 países con una capacidad de generación instalada de $1.292 \mathrm{GW}$. Entre estos países se destaca China con una capacidad de generación instalada de $352,26 \mathrm{GW}$ y una generación anual de 1.232.900 GWh [3]. Le siguen en importancia Brasil con $104 \mathrm{GW}$ de potencia instalada y Estados Unidos con $103 \mathrm{GW}$.

La energía hidráulica presenta numerosas ventajas en comparación con las fuentes fósiles y también con respecto a otras fuentes renovables. Respecto a las fuentes fósiles tienen la ventaja de la reducción de contaminación, principalmente la reducción en las emisiones de $\mathrm{CO}_{2}$ que es la principal causa del efecto invernadero [4]. Otra ventaja es la reducción en los costos operativos de generación, debido a que esta tecnología no se encuentra sujeta a los costos de los combustibles fósiles.

Esta característica vuelve a las centrales hidroeléctricas muy convenientes una vez que sus costos de instalación han sido cubiertos. Con respecto a otras fuentes renovables, la hidráulica presenta una mayor facilidad para organizar su programación en virtud a que se puede hacer una mejor previsión de su disponibilidad. En cambio, las fuentes eólicas y solares fotovoltaicas se encuentran limitadas principalmente por su intermitencia [5]. Como consecuencia, esta 
intermitencia obliga a tener una mayor cantidad de sistemas de generación de reserva o de almacenamiento de energía para estas tecnologías [6], [7].

No obstante, la operación de una planta hidráulica es una tarea muy compleja, y el hecho de no hacerlo de una manera eficiente puede originar una pérdida económica de enormes cantidades [6]. La programación matemática es una poderosa herramienta que se aplica para la toma de decisiones en numerosos procesos industriales. Su objetivo es obtener la solución más conveniente dentro de un grupo de alternativas posibles, teniendo en cuenta los recursos de los cuales se dispone. Gracias a los resultados obtenidos con la aplicación de estas herramientas, los modelos de optimización han cobrado gran relevancia en el campo de los sistemas de potencia eléctrica [8], [9]. En este grupo se incluye la operación de centrales hidroeléctricas.

El problema matemático que consiste en determinar la programación óptima de unidades generadoras hidráulicas es conocido como Problema de Asignación de Unidades Hidráulicas (AUH, [10]). Los modelos matemáticos que resuelven este problema han aumentado su complejidad en los últimos 10 años ([11]). En [12] se presenta un modelo estocástico que permite la coordinación entre dos niveles de decisión a través de un método basado en la Descomposición de Benders, el escenario de pruebas es el sistema eléctrico de Brasil que mezcla varias fuentes. Sin embargo, con esta técnica, en ciertos casos sólo se pueden encontrar mínimos locales, mientras que en otros casos ni siquiera se puede lograr la convergencia con el óptimo local. En [13] se presenta un análisis entre distintos métodos de programación matemática utilizados para resolver el problema AUH, en el mismo se comparan distintas consideraciones, objetivos y restricciones.

Cuando se representa el funcionamiento de una unidad hidroeléctrica mediante un modelo matemático, se advierte que existe una relación no lineal entre la potencia generada, el caudal turbinado y el salto hidráulico. Esto provoca que cuando se consideran las restricciones originales de este problema, se obtiene un modelo del tipo de Programación Mixta Entera No Lineal (PMENL). La principal desventaja de este tipo de modelos es su elevado requerimiento de procesamiento para ser resuelto, más aún cuando se trata de sistemas eléctricos a gran escala [14]. Frente a este inconveniente, en los últimos años se ha desarrollado una serie de técnicas de linealización que permiten aproximar estos problemas con suficiente nivel de precisión. De esta forma se obtienen modelos matemáticos del tipo de Programación Mixta Entera Lineal (PMEL). Estos modelos tienen como principales características un menor esfuerzo computacional, el alcance del óptimo global, y la flexibilidad para anexar nuevas restricciones [15]. En este contexto, los autores de [16] presentan un modelo PMEL donde se resuelve la programación de un sistema eléctrico donde existen 2 centrales hidroeléctricas, que además tienen la capacidad de bombeo. Sin embargo, en este trabajo no se consideran los efectos del salto hidráulico, el cual se define como la diferencia entre las cotas de agua de los reservorios superiores e inferiores de la central hidráulica. Esto puede dar origen a resultados alejados de la realidad porque no consideran los efectos de esta variación en las turbinas. En [17] se presenta un modelo PMEL donde sí se considera este efecto mediante la introducción de variables binarias, y el caso de estudio es una planta con 5 unidades y $3.000 \mathrm{MW}$ de potencia total instalada. Además, en [18] también se presenta un modelo de este tipo, el cual tiende a reducir la diferencia entre picos de consumo. Todos estos modelos PMEL tienen en común que no contemplan la posibilidad de operar las descargas de forma independiente, tanto por compuertas como por turbinado.

Itaipú es la central hidroeléctrica más grande del mundo, en cuanto a producción de electricidad (récord de generación con 103,1 TWh en 2016), y la segunda en cuanto a capacidad instalada (14.000 MW). Es un ente binacional que produce el 90\% de la electricidad consumida en Paraguay y el 15\% de Brasil. Desde su puesta en funcionamiento en 1.984, ha generado más de 2,6 billones de MWh [19]. Cuenta con 20 unidades generadoras del tipo turbina Francis con una potencia nominal de $715 \mathrm{MW}$ cada una. Su embalse tiene un volumen de $29.000 \mathrm{Hm}^{3}$ con el mejor índice de aprovechamiento de agua de Brasil. El río Paraná, el octavo con mayor extensión a nivel mundial con casi $4.900 \mathrm{~km}$, hace el principal aporte a la central y divide los territorios de los dos países que la operan. El vertedero es del tipo de ladera, se encuentra ubicado a la margen derecha de la sala de máquinas y su capacidad máxima de descarga es de $62.200 \mathrm{~m}^{3} / \mathrm{s}$. La Fig. 1 muestra la ubicación de la central y su embalse en el mapa del continente americano. La generación de esta central se transmite hacia los consumidores de los países de Paraguay y Brasil, cuyas características como mercados eléctricos latinoamericanos son estudiados en [20]. En lo relativo a la transmisión de la electricidad generada, esta etapa se encuentra descripta y formulada en [21].

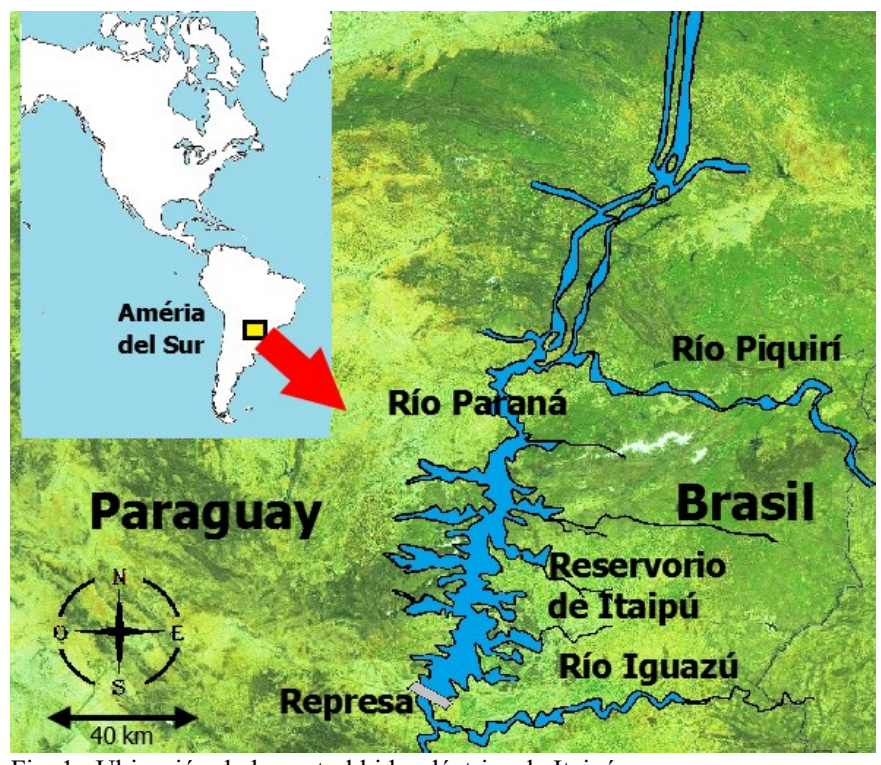

Fig. 1. Ubicación de la central hidroeléctrica de Itaipú.

En vista de lo anterior, este trabajo presenta dos aportes fundamentales. 1) El primero de ellos es presentar un modelo PMEL para la operación de centrales hidroeléctricas, teniendo en cuenta varios aspectos que permiten obtener soluciones más reales. Se toman algunas guías presentadas en [22], pero este ha sido modificado para aplicarse a un modelo a gran escala, sin el modo de bombeo, lo cual repercute en la operación de la planta y en los balances del embalse. Además, 
esta nueva formulación permite individualizar y operar las compuertas de descarga de la central, hecho que no se considera a menudo. Obteniendo así, soluciones más cercanas al caso de estudio.

2) El segundo aporte es el estudio de manera integral de la central hidroeléctrica Itaipú, utilizando el modelo propuesto. La importancia de la central es vital no solo para el binomio Paraguay-Brasil, sino para toda Sudamérica, dada su capacidad de generación. Se estudiará la aplicación del nuevo modelo de este sistema para la programación diaria de la central utilizando datos reales, considerando la configuración más reciente de 20 unidades. Esto afectará no solo a los resultados, sino también a la forma de operación de la central, comparada con configuraciones anteriores ([23]).

El resto del trabajo se organiza de la siguiente manera: la Sección III presenta el modelo matemático a ser resuelto, la Sección IV introduce los escenarios que se estudiarán, la Sección V compara los resultados y la Sección VI remarca las conclusiones.

\section{MODELO MATEMÁTICO}

En esta sección se presenta un modelo matemático que permite resolver el problema AUH para la operación de una central a gran escala. Para esto se presentan el modelo original y las técnicas de linealización aplicadas.

\section{A. Operación de una Central Hidráulica-Modelo Clásico}

Según [24], [25] la función objetivo maximizará el beneficio total de la central, lo que incluye la reducción de los costos asociados para un horizonte de programación de 24 horas. Donde $i$ es el índice para cada equipo turbina, $t$ es el índice para el período de tiempo, $I$ es el número total de equipos, $T$ es el horizonte de programación, $p_{i, t}$ es la potencia generada (MW), y $\rho_{i}^{p p}$ es el precio horario de generación para cada tiempo $t$. Además $\sigma_{i}$ es el costo de la potencia unitaria producida (USD/MW), $S U_{i, t} / S D_{i, t}$ es una variable binaria que vale 1 cuando la unidad se enciende/apaga, y $c_{i}^{S U} / c_{i}^{S D}$ es el costo de encendido/apagado de la unidad (USD).

$$
\begin{aligned}
\max \text { profit }= & \sum_{i=1}^{I} \sum_{t=1}^{T}\left[\rho_{i}^{p p} p_{i, t}-p_{i, t} \sigma_{i}-S U_{i, t} c_{i}^{S U}\right. \\
& \left.-S D_{i, t} c_{i}^{S D}\right]
\end{aligned}
$$

Una serie de restricciones limitan el problema AUH, la principal de ellas es la satisfacción de la demanda de electricidad, la cual se representa en (2).

$$
\sum_{i=1}^{I} p_{i, t} \geq D_{t} \quad, \quad t=1, \ldots, T
$$

Donde $D_{t}$ es la demanda de electricidad requerida por el operador del sistema. Dicha demanda es pactada previamente, y si la central puede generar una cantidad mayor a dicha demanda puede vender el excedente en el mercado eléctrico, según convenga en base al precio horario de la electricidad.
La potencia generada por cada unidad (en MW) de la central se determina según (3), en base a lo descripto en [26].

$$
\begin{aligned}
& p_{i, t}=g p \delta_{i, t} S_{i, t} \mu_{i}^{g e} \mu_{i}^{t u} \mu_{i}^{c p} \mu_{i}^{t r} /\left(1 * 10^{6}\right) \\
& i=1, \ldots, I ; t=1, \ldots, T
\end{aligned}
$$

Siendo $g$ la gravedad $\left(9,8 \mathrm{~m} / \mathrm{s}^{2}\right), p$ el peso específico del agua $\left(1.000 \mathrm{~kg} / \mathrm{m}^{3}\right), \quad \delta_{i, t}$ la variable del caudal turbinado $\left(\mathrm{m}^{3} / \mathrm{s}\right), S_{i, t}$ es una variable de salto hidráulico $(\mathrm{m})$. En cuanto a las constantes, según [27], $\mu_{i}^{g e}$ es el rendimiento del generador eléctrico (entre 0,92 y 0,97$), \quad \mu_{i}^{t u}$ es el rendimiento de la turbina hidráulica (entre 0,75 y 0,94$), \mu_{i}^{c p}$ es el rendimiento mecánico del acoplamiento turbina-alternador (entre 0,95 y $0,99)$, y $\mu_{i}^{t r}$ es el rendimiento del transformador de salida $(0,9)$. Esta restricción implica el producto entre dos variables continuas $\mathrm{y}$, esto complejiza la resolución del problema de optimización.

Las ecuación (4) determina el volumen del embalse superior. En este caso participan las variables de caudales turbinados y las constantes $r f_{\text {in }} / r f_{\text {out }}$ que corresponden a los caudales de los ríos entrantes y salientes al embalse. $e_{t-1}$ es el volumen de agua en el período de tiempo anterior. Esta restricción se basa en trabajos como [28]. Otros modelos como [29] consideran en esta restricción variables de filtraciones. Pero dichas variables se consideran despreciables a los efectos de los casos de estudio del presente trabajo.

$$
\begin{aligned}
& e_{t}=e_{t-1}+r f_{\text {in }}-r f_{\text {out }}-\sum_{i=1}^{I} \delta_{i, t}, \\
& t=1, \ldots, T
\end{aligned}
$$

La restricción (5) establece que al final del horizonte de programación el volumen del embalse debe ser igual o mayor al nivel del comienzo. Esto asegura la reserva de energía. Frente a situaciones extremas o imprevistas (como diferencias entre el volumen de agua provisto y el real) esta restricción puede ser modificada y/o eliminada según el caso.

$e_{t=T} \geq e_{t=1}$

\section{B. Mejoras al Modelo Clásico - Linealización de Restricciones}

En la Fig. 2 se puede observar la representación de la gráfica que relaciona la potencia producida, con el caudal descargado, y el salto hidráulico de una turbina de la Central Itaipú (con base a los datos de [23] y [19]). La regla general es que la potencia generada depende del caudal turbinado y el salto hidráulico. A mayor caudal y mayor salto, mayor potencia producida. Sin embargo, en la realidad existen excepciones como se observa en la figura. Existen niveles de descarga y salto en donde los coeficientes de eficiencia son máximos. $\mathrm{Y}$ existen otros niveles por donde el rendimiento cae, dicha caída puede ser tal que hasta en ocasiones puede 
ocurrir que a mayor descarga o mayor salto, la potencia no aumente. De aquí la importancia de las curvas de operación.

Se observa que el máximo rendimiento de la turbina se obtiene cuando su salto hidráulico se encuentra cerca de los $118 \mathrm{~m}$, y por debajo de los $115 \mathrm{~m}$ la eficiencia disminuye de manera considerable.

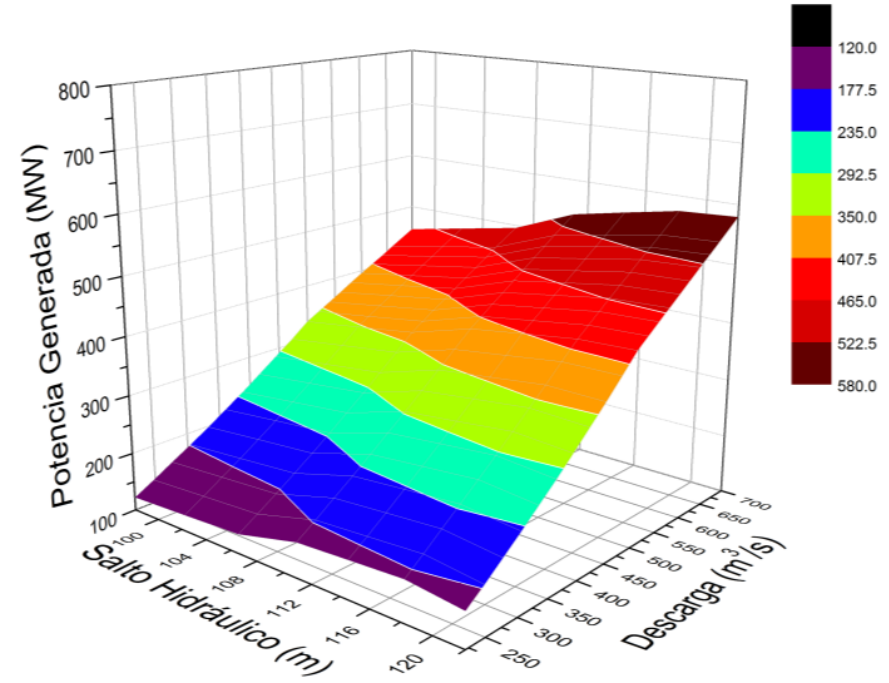

Fig. 2. Curva Generación-descarga para una turbina de la central Itaipú.

Para lograr la linealización de estas curvas, se recurre a una serie de restricciones. La restricción (6) establece que la variable binaria $z_{i, t}$, que representa el estado de funcionamiento de la unidad, es igual a la sumatoria de las variables de peso $\phi_{s s, g s, i, t}$. La variable de estado equivale a 1 cuando la unidad genera electricidad, y 0 en otro caso. Siendo $g s$ el índice para cada segmento de generación y $s s$ el índice para cada nivel de salto hidráulico. A su vez, $G S$ y $S S$ son los números totales de segmentos de generación y de niveles de salto hidráulico.

$\sum_{s S=1}^{S S} \sum_{g s=1}^{G S} \phi_{s s, g s, i, t}=z_{i, t}, \quad i=1, \ldots, I ; t=1, \ldots, T$

La restricciones (7) y (8) relacionan el volumen del embalse con la variable binaria $S H_{s s, t}$ la cual vale 1 cuando el nivel del embalse coincide con el nivel de salto correspondiente, y de lo contrario vale 0 . Mientras $v_{s S}^{\min } / v_{s s}^{\max }$ es una constante que representa el nivel mínimo/máximo de volumen que pertenece al salto $s s$.

$\sum_{s s=1}^{S S} S H_{s s, t} v_{s s}^{\min } \leq e_{t}, t=1, \ldots, T$
$e_{t} \leq \sum_{s s=1}^{S S} S H_{s s, t} v_{s s}^{\max }, t=1, \ldots, T$

Las restricciones (9) y (10) establecen el valor del salto hidráulico $S_{i, t}$ para cada turbina $i$ relacionando el mismo con la variable de peso $\phi_{s s, g s, i, t}$. Donde $S_{s s}^{\max } / S_{s s}^{\min }$ son constantes que corresponden al máximo/mínimo valor de salto para el nivel de salto $s s$, y $\Gamma$ es el valor máximo posible de salto hidráulico.

$S_{i, t} \leq \sum_{g s=1}^{G S} \sum_{s S=1}^{S S} \phi_{s S, g s, i, t} S_{s S}^{\max }+\Gamma\left(1-z_{i, t}\right)$

$i=1, \ldots, I ; t=1, \ldots, T$

$S_{i, t} \geq \sum_{g s=1}^{G S} \sum_{S S=1}^{S S} \phi_{s s, g s, i, t} S_{s S}^{\min }$

$i=1, \ldots, I ; t=1, \ldots, T$

Las restricciones (11) y (12) definen el valor de las variables correspondientes a potencia generada $p_{i, t}$ y caudal descargado $\delta_{i, t} . \Delta p_{s s, g s, i}$ y $\Delta \delta_{s s, g s, i}$ corresponden al valor del punto de muestra de potencia y caudal para los segmentos $s s, g s$ y la unidad $i$.

$$
\begin{aligned}
& p_{i, t}=\sum_{g s=1}^{G S} \sum_{s s=1}^{S S} \phi_{s s, g s, i, t} \Delta p_{s s, g s, i} \\
& i, \ldots, I ; t=1, \ldots, T \\
& \delta_{i, t}=\sum_{g s=1}^{G S} \sum_{s s=1}^{S S} \phi_{s s, g s, i, t} \Delta \delta_{s s, g s, i} \\
& i=1, \ldots, I ; t=1, \ldots, T
\end{aligned}
$$

A su vez las unidades tienen valores máximos y mínimos de operación debido a razones técnicas y de seguridad. Es por eso que la restricción (13) determina estos límites. Donde $\overline{p_{\imath}} / \underline{p_{i}}$, son los límites máximos/mínimos de generación para la unidad.

$\underline{p_{i}} z_{i, t} \leq p_{i, t} \leq z_{i, t} \overline{p_{l}}, \quad i=1, \ldots, I ; t=1, \ldots, T$

La restricción (14) establece que la variable binaria que representa los posibles niveles de salto puede estar activa para un solo nivel en cada período de tiempo.

$$
\sum_{S S=1}^{S S} S H_{S S, t}=1, \quad t=1, \ldots, T
$$

En (15) se relaciona el nivel del embalse con las variables de peso para la generación $\phi_{s s, g s, i, t}$.

$$
\sum_{g s=1}^{G S} \sum_{i=1}^{I} \phi_{S S, g s, i, t} \leq S H_{s s, t}, \quad s s=1, \ldots, \mathrm{SS} ; t=1, \ldots, T
$$

En las restricciones (16) y (17) se relaciona la variable binaria $S H_{s s, t}$ con la determinación del salto hidráulico. En este caso $B$ es un número positivo lo suficientemente grande. 
$\sum_{i=1}^{I} S_{i, t}-B\left(1-\sum_{s s=1}^{S S} S H_{s s, t}\right) \leq S H_{s s, t} S_{s s}^{\max }$

$t=1, \ldots, T$

$$
\sum_{\substack{i=1 \\ t=1, \ldots, T}}^{I} S_{i, t}+B\left(1-\sum_{s s=1}^{S S} S H_{s s, t}\right) \geq S H_{s s, t} S_{s S}^{\min }
$$

La restricción (18) determina los valores de las variables binarias $S U_{i, t} / S D_{i, t}$ que valen 1 cuando la unidad se enciende/apaga en el momento $t$. Y la restricción (19) establece que solo una puede estar activa, en un mismo período.

$z_{i, t}-z_{i, t-1}=S U_{i, t}-S D_{i, t}, i=1, \ldots, I ; t=1, \ldots, T$

$S U_{i, t}+S D_{i, t} \leq 1, \quad i=1, \ldots, I ; t=1, \ldots, T$

Las restricciones (9-19) reemplazan a la restricción (3). Esto se hace en virtud de reducir el requerimiento computacional. La restricción (20) representa el volumen de agua del embalse. Esta restricción se diferencia de las del modelo clásico porque considera la evacuación de agua mediante las compuertas. Por ello, $\delta v_{c, t}$ representa la variable de descarga por compuertas, mientras que $c$ es el índice de cada compuerta. Esta restricción brinda un grado de acercamiento mayor con la realidad que los modelos clásicos.

En efecto, la mayoría de los modelos no contemplan que las compuertas puedan ser operadas de manera independiente a la generación. En dichos escenarios, las únicas formas consideradas en que puede reducirse el volumen del embalse son mediante el caudal "turbinado" o mediante el filtrado. Pero estos caudales son muy distintos a los que circulan por las compuestas. Es por esto que el modelo propuesto brinda mejores posibilidades para operar la central.

$e_{t}=e_{t-1}+r f_{\text {in }}-r f_{\text {out }}-\sum_{i=1}^{I} \delta_{i, t}-\sum_{c=1}^{C} \delta v_{c, t}$,

$t=1, \ldots, T$

\section{ESCENARIOS DE ESTUdIO}

Para probar la efectividad del modelo PMEL propuesto se utiliza el sistema que pertenece a la Central de Itaipú. El área abarcada tiene más de $1.350 \mathrm{~km}^{2}$, con una extensión de 170 $\mathrm{km}$ y un ancho máximo de $12 \mathrm{~km}$. La central posee 20 turbinas del tipo Francis y 14 compuertas para regular el embalse.

Los datos relativos a la generación y descarga para cada una de las turbinas se presentan en la Tabla I. Los tres niveles de generación descargan 220, 500 y $700 \mathrm{~m}^{3} / \mathrm{s}$ por turbina, respectivamente, a su vez se consideran tres niveles de salto.

Los datos relativos al embalse se encuentran en la Tabla II. La Tabla III presenta los precios horarios pagados por generación para las 24 horas del horizonte de programación, se usan los mismos precios para todos los escenarios estudiados.

TABLA I

POTENCIA GENERADA POR TURBINA. EN (MW)

\begin{tabular}{cccc}
\hline \hline $\begin{array}{c}\text { Segmento } \\
\text { de } \\
\text { Generación }\end{array}$ & 98 & Salto Hidráulico (m) \\
\hline A & 121,94 & 111 & 122 \\
\hline B & 332,56 & 398,37 & 157,87 \\
C & 455,24 & 539,07 & 423,21 \\
\hline \hline
\end{tabular}

TABLA II

\begin{tabular}{|c|c|}
\hline Característica & Valor \\
\hline Volumen operativo mínimo $\left(\mathrm{Hm}^{3}\right)$ & 5.000 \\
\hline Volumen operativo máximo $\left(\mathrm{Hm}^{3}\right)$ & 29.000 \\
\hline Volumen inicial $\left(\mathrm{Hm}^{3}\right)$ & 19.601 \\
\hline Caudal normal del río $\left(\mathrm{Hm}^{3} / \mathrm{h}\right)$ & 40,68 \\
\hline Min. vol. para el nivel salto $1\left(\mathrm{Hm}^{3}\right)$ & 5001 \\
\hline Min. vol. para el nivel salto $2\left(\mathrm{Hm}^{3}\right)$ & 12.667 \\
\hline Min. vol. para el nivel salto $3\left(\mathrm{Hm}^{3}\right)$ & 19.001 \\
\hline Max. vol. nivel Salto $1\left(\mathrm{Hm}^{3}\right)$ & 12.666 \\
\hline Max. vol. nivel Salto $2\left(\mathrm{Hm}^{3}\right)$ & 19.000 \\
\hline Max. vol. nivel Salto $3\left(\mathrm{Hm}^{3}\right)$ & 29.000 \\
\hline Max. descarga por compuertas $\left(\mathrm{Hm}^{3} / \mathrm{h}\right)$ & 216,72 \\
\hline
\end{tabular}

DATOS DEL EMBALSE

TABLA III

PRECIO EN USD POR MW GENERADO

\begin{tabular}{cccl}
\hline \hline Hora & $\rho_{i}^{p p}$ & Hora & $\rho_{i}^{p p}$ \\
\hline 1 & 59,57 & 13 & 69,015 \\
2 & 54,23 & 14 & 68,605 \\
3 & 56,69 & 15 & 66,140 \\
4 & 57,10 & 16 & 65,934 \\
5 & 56,49 & 17 & 66,756 \\
6 & 56,69 & 18 & 72,713 \\
7 & 57,51 & 19 & 72,71 \\
8 & 60,80 & 20 & 78,26 \\
9 & 62,03 & 21 & 78,87 \\
10 & 65,73 & 22 & 78,67 \\
11 & 66,14 & 23 & 78,07 \\
12 & 66,55 & 24 & 76,00 \\
\hline \hline
\end{tabular}

\section{ANÁlisis DE RESULTADOS Y COMPARACIONES}

Para poder probar el modelo de manera exhaustiva, se estudiaron tres escenarios. En el Escenario 1 el embalse se encuentra en un nivel medio-alto, frente a un caudal normal del río. El Escenario 2, tiene un nivel de embalse máximo y 
con alto caudal del río, donde se requiere evacuar el exceso por las compuertas. Por último el Escenario 3 tiene un nivel bajo en el embalse y un pobre caudal del río. Para los tres escenarios, se considerará que la central puede vender toda la generación que produzca en el mercado a precio horario.

El modelo se prueba en el software GAMS con el resolvedor lineal CPLEX [30] para un GAP relativo de 0.00001 . EL GAP relativo se define como la diferencia que surge entre el valor de la mejor solución conocida, y el valor de la mejor solución posible dividido el mejor límite.

\section{A. Escenario 1-Nivel Medio de Caudal}

El modelo está compuesto por 7.302 ecuaciones, 9.011 variables y 1.464 variables binarias. CPLEX resolvió el problema en 4,469 segundos, obteniendo un beneficio total de USD 11.341.567.

La Fig. 3 presenta, en el eje vertical izquierdo, el perfil de generación de la central teniendo en cuenta la potencia producida por cada una de los 20 grupos turbina, asumiendo que el total de los mismos se encuentra disponible. Además en el eje vertical derecho se ilustra la evolución del precio horario de generación. El principal limitante de la generación son las características técnicas del embalse, lo cual hace que para obtener el mejor beneficio posible, sea mayor la generación en los horarios con mayores precios de generación.

La generación total durante todo el horizonte de programación es de 262.947 MWh. Solo un generador está funcionando las 24 horas de programación, el 15. Los restantes funcionan 18 horas. No hay motivos específicos para la elección del generador 15 por sobre el resto, debido a la simetría del problema en cuanto a esta característica.

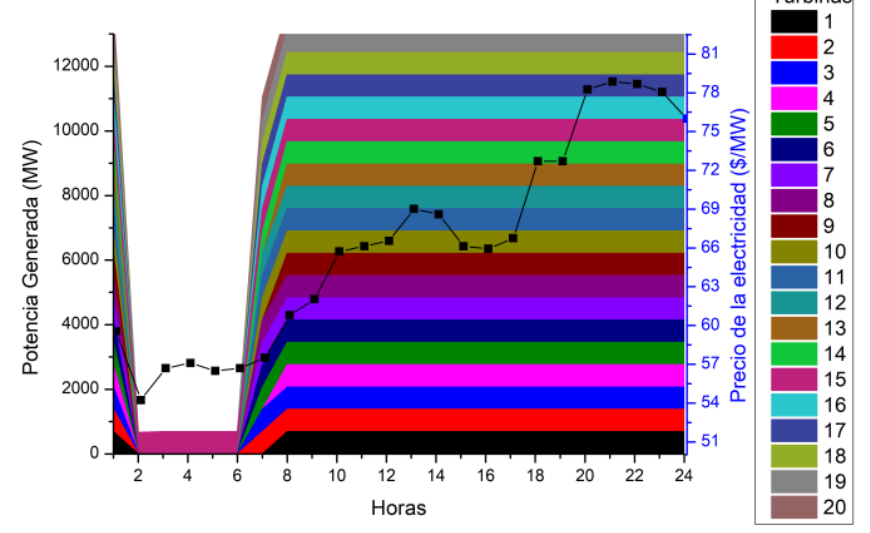

Fig. 3. Perfil de generación vs. precios de la energía. Escenario 1.

En cuanto al volumen del embalse de la central, se puede observar su evolución durante todo el horizonte de programación en el eje vertical izquierdo de la gráfica de la Fig. 4. A su vez, el valor del precio pagado por generación se observa en el eje vertical derecho de la gráfica. El valor del volumen del embalse comienza en la hora 1 según las condiciones iniciales, y va aumentando hasta llegar a un máximo de $19.790 \mathrm{Hm}^{3}$ entre las horas 6 y 7. El promedio de ingreso al embalse es de $40,68 \mathrm{Hm}^{3} / \mathrm{h}$ y el promedio descargado es de $41,15 \mathrm{Hm}^{3} / \mathrm{h}$. Se puede observar que este incremento del volumen coincide con el intervalo de tiempo donde el precio pagado por generación es menor.

Posteriormente, el nivel de potencia generada aumenta, y por consiguiente aumenta la descarga de agua a través de las tuberías. Al final del horizonte de programación, el volumen del embalse debe ser mayor o igual al valor del comienzo del horizonte. Esto es debido a la imposición de la restricción (5), y constituye una reserva de energía del sistema. En la gráfica también se observan los tres niveles de salto hidráulico previstos, en ese caso el volumen del embalse se mantiene entre los límites correspondientes al nivel 3. Para que el valor del volumen ingrese a la zona de los niveles 1 o 2 , debe eliminarse temporalmente la restricción (5) y esto se aplica en épocas de sequías cuando el caudal que aporta el río es menor.

Es importante mencionar que para este escenario, como el nivel del caudal del río es normal, no se ha producido la apertura de ninguna compuerta y por eso el caudal descargado a través de ellas es cero.

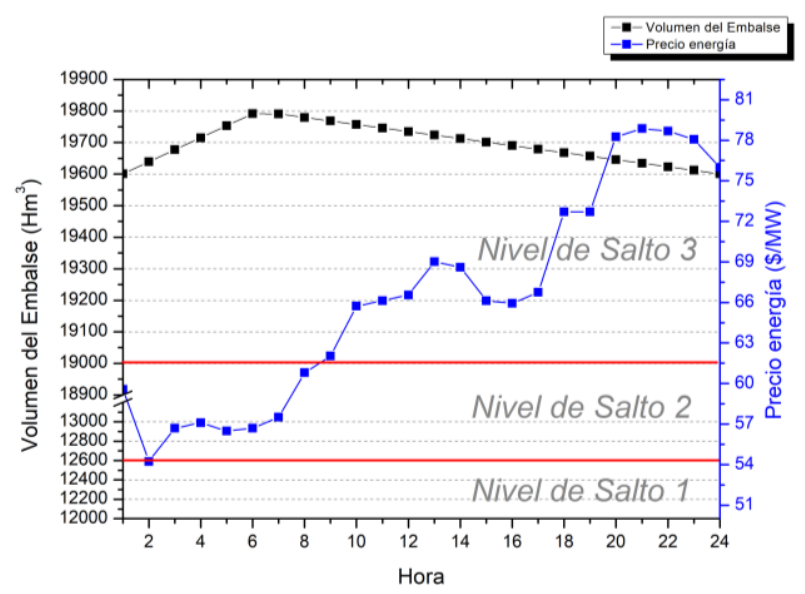

Fig. 4. Volumen de embalse vs. precios de la energía. Escenario 1.

\section{B. Escenario 2 - Nivel Alto de Caudal}

Para el Escenario 2, se establece una situación donde el nivel del embalse está cerca del máximo, y el nivel del caudal del río es un $50 \%$ más de lo normal, para una época de grandes lluvias y crecientes. Por lo tanto, el nivel inicial del embalse es de $28.800 \mathrm{Hm}^{3}$, y el caudal del río es de 16.967 $\mathrm{m}^{3} / \mathrm{s}$. En este escenario el valor de la función objetivo es de USD 13.560.156, y el tiempo de resolución es de 4,5 segundos.

La evolución del embalse y los caudales descargados se puede observar en la Fig. 5. En este escenario, se advierte que el embalse se encuentra cerca del máximo, por lo tanto, la central se encuentra turbinando agua al máximo nivel posible.

Con un promedio de descarga de $51,84 \mathrm{Hm}^{3} / \mathrm{h}$, suma un total de $1.244,16 \mathrm{Hm}^{3}$ turbinados durante todo el horizonte de programación. Cada generador produce cada hora 690,2 MWh. Dado el excesivo caudal de ingreso del río al embalse, es necesaria una apertura de las compuertas, porque no basta el caudal que escapa por las tuberías de las veinte turbinas. Es por eso que entre las horas señaladas en la figura, alguna de las 14 compuertas se abre para descomprimir el embalse. En cuanto al volumen del embalse se observa una tendencia al crecimiento hasta la hora 8 , cuando el volumen cae debido a la 
apertura de las compuertas. Posteriormente, comienza otra tendencia de crecimiento hasta la hora 17 , para caer $\mathrm{y}$, por tanto, alcanza el nivel de $29.000 \mathrm{Hm}^{3}$.

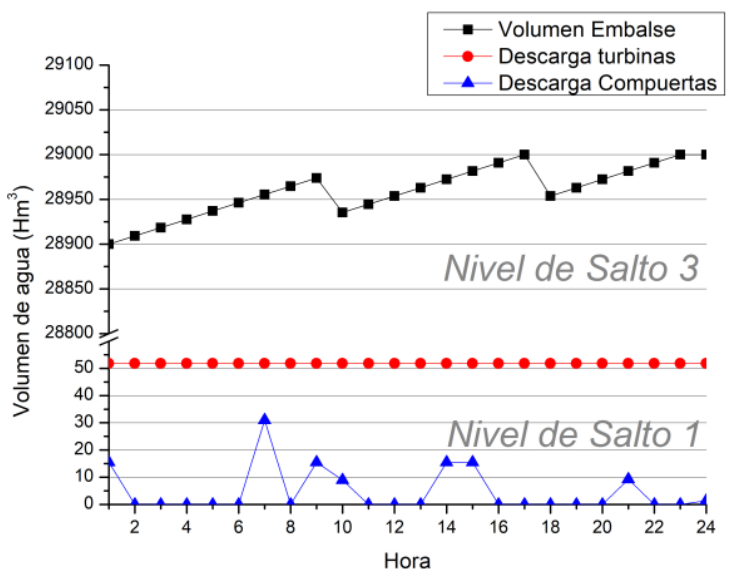

Fig. 5. Volumen embalse y caudales turbinados y descargados, Escenario 2.

\section{Escenario 3 - Nivel Bajo de Caudal}

El Escenario 3 corresponde a una situación donde el nivel del embalse está bajo, y el caudal del río es cercano a la mitad del valor normal. Esto corresponde a una época con escasez de lluvias y de sequías. Por lo tanto, el nivel inicial del embalse es de $16.600 \mathrm{Hm}^{3}$, y el caudal del río es de $5.650 \mathrm{~m}^{3} / \mathrm{s}$. El beneficio total es de USD 6.429.801, con un tiempo de procesamiento de 35,8 segundos. La Fig. 6 muestra la producción de cada una de las turbinas.

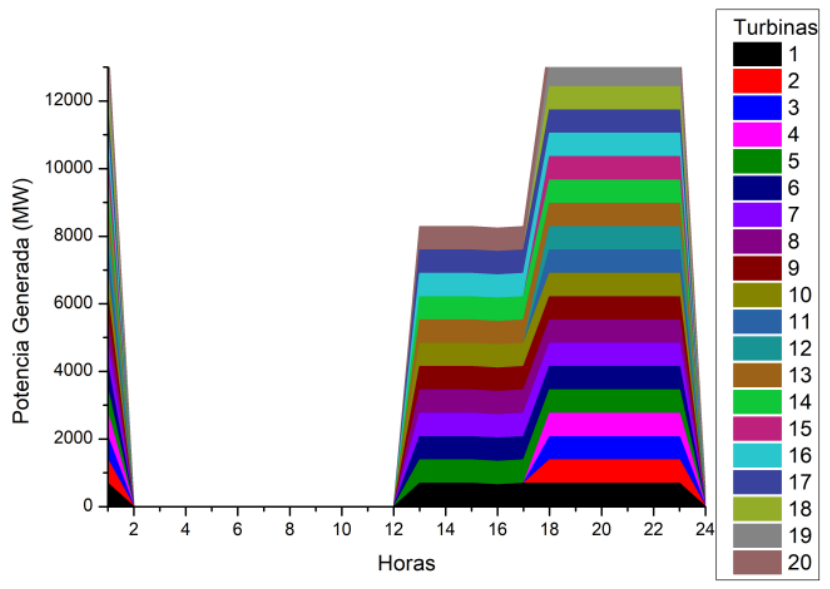

Fig. 6. Perfil de generación de las veinte unidades. Escenario 3.

La razón de evolución del embalse y también los caudales descargados se presentan en la Fig. 7. Como el embalse se encuentra en un nivel bajo, la central turbina agua en menor medida que los escenarios anteriores.

El promedio de descarga a través de las turbinas es de 21.7 $\mathrm{Hm}^{3} / \mathrm{h}$, y la suma durante todo el horizonte de programación es de $520 \mathrm{Hm}^{3}$. Como el caudal de ingreso del río al embalse es bajo, no existe apertura alguna de las compuertas. La elección del orden en que los generadores se encienden, encuentra su fundamento en la resolución de un problema de simetría. Si se desea un orden de encendido específico hay que anexar más restricciones al problema.

El volumen del embalse tiene una tendencia creciente que persiste las primeras 12 horas hasta llegar a un valor de 12.743 $\mathrm{Hm}^{3}$. A partir de la hora 13 comienza una tendencia decreciente con pendiente reducida hasta la hora 23 , donde la pendiente decreciente aumenta. En la figura también se puede advertir que la evolución del volumen del embalse implica un cambio en el nivel de salto hidráulico. A la hora 6 hay un cambio en el nivel de salto seleccionado, pasando del nivel de salto 2 al nivel 3. Mientras que a la hora 21, hay un cambio de nivel 3 a nivel 2 .

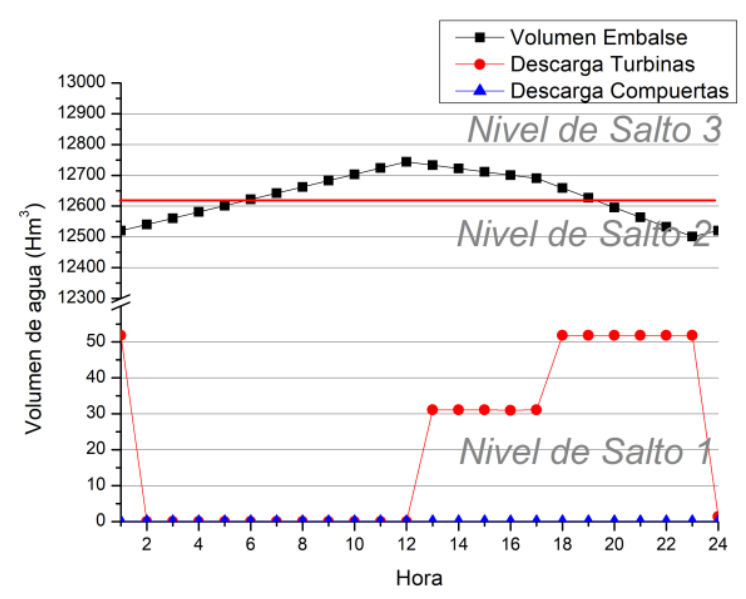

Fig. 7. Volumen embalse y caudales turbinados y descargados, Escenario 3.

\section{CONClusiones}

El presente trabajo introduce un nuevo modelo de Programación Mixta Entera Lineal (PMEL), mediante el cual se logra la operación de una central hidráulica de gran escala. Las restricciones que modelan la generación son no-lineales y cuando se intenta resolver el modelo con las restricciones originales, esto puede resultar imposible o requerir mucho tiempo de procesamiento. Debido a este inconveniente, se introduce una técnica de linealización para las curvas de generación de cada una las turbinas. Este nuevo modelo se diferencia en que considera la apertura individual de cada compuerta en el balance de cantidad de agua en el embalse, permitiendo obtener resultados más cercanos a la realidad.

Se estudia la Central Hidráulica de Itaipú, que tiene el record mundial de ser la central con mayor cantidad de electricidad generada. Para saber el comportamiento del sistema frente a situaciones extremas es que se estudian tres escenarios: con nivel de caudales de ingreso al embalse normal, con caudales elevados y con caudales bajos. Cuando se intenta resolver la operación de la central para un horizonte de programación de 24 horas, con el modelo original no-lineal, no pueden obtenerse soluciones factibles en un tiempo de 3.600 segundos. Pero cuando se resuelven los problemas con el modelo propuesto, se obtiene una solución factible que satisface la tolerancia en un tiempo bajo. El tamaño de los problemas supera las 7.300 ecuaciones y las 1.400 variables binarias. Del análisis de los tres escenarios planteados se puede concluir que la central opera tratando de conservar la 
disponibilidad de agua para que sea utilizada en la generación de electricidad en momentos cuando el beneficio económico es mayor. En escenarios extremos, la central también opera el comando de compuertas para velar por la seguridad de la estructura del embalse. Se observa en el Escenario 2 que el beneficio económico no aumenta de manera proporcional al aumento del volumen del embalse, como consecuencia de la eficiencia de las curvas de operación.

Es importante mencionar que el Escenario 3 se ha planteado para observar cambios en los niveles de saltos hidráulicos. Estos cambios generalmente se manifiestan de manera más notable en la programación a largo plazo. Es por este motivo que los valores de los escenarios han sido seleccionados para evidenciar particularidades que pueden ocurrir en ciertas circunstancias.

\section{AgradeCIMIENTOS}

El autor agradece al Consejo Nacional de Investigaciones Científicas y Técnicas (CONICET) por el soporte recibido.

\section{REFERENCIAS}

[1] International Energy Agency, "Key World Energy Statistics 2018," 2018.

[2] E. M. Rana Adib, Hannah E. Murdock, Fabiani Appavou, Adam Brown, , Anna Leidreiter, Christine Lins, Hannah E. Murdock, Evan Musolino, Ksenia Petrichenko, Timothy C. Farrell, Thomas Thorsch Krader, , Janet L. Sawin , Kristin Seyboth, Jonathan Skeen, , Freyr S, "Renewables 2016: Global Status Report," 2016.

[3] International Hydropower Association, "2019 Hydropower Status Report," 2019.

[4] M. Caballero, S. Lozano, and B. Ortega, "Efecto invernadero, calentamiento global y climático," Rev. UNAM, p. 11, 2007.

[5] B. K. Sovacool, "The intermittency of wind, solar, and renewable electricity generators: Technical barrier or rhetorical excuse?," Util. Policy, vol. 17, no. 3-4, pp. 288-296, 2009.

[6] S. Xia, K. W. Chan, X. Luo, S. Bu, Z. Ding, and B. Zhou, "Optimal sizing of energy storage system and its cost-benefit analysis for power grid planning with intermittent wind generation," Renew. Energy, vol. 122, pp. 472-486, 2018.

[7] M. Baumann, M. Weil, J. F. Peters, N. Chibeles-Martins, and A. B. Moniz, "A review of multi-criteria decision making approaches for evaluating energy storage systems for grid applications," Renewable and Sustainable Energy Reviews. pp. 516-534, 2019.

[8] J. A. Momoh, Electric Power System Applications of Optimization, Second Edition. 2017.

[9] S. M. Naeem Nawaz and S. Alvi, "Energy security for socioeconomic and environmental sustainability in Pakistan," Heliyon, vol. 4, no. 10, 2018.

[10] Chao-An Li, A. J. Svoboda, Chung-Li Tseng, R. B. Johnson, and E. Hsu, "Hydro unit commitment in hydro-thermal optimization," IEEE Trans. Power Syst., vol. 12, no. 2, pp. 764-769, May 1997.

[11] G. W. Chang et al., "ExperiencesWith Mixed Integer Linear Programming Based Approaches on Short-Term Hydro Scheduling," IEEE Trans. Power Syst., vol. 16, no. 4, pp. 743-749, 2001.

[12] E. C. Finardi, R. D. Lobato, V. L. de Matos, C. Sagastizábal, and A. Tomasgard, "Stochastic hydro-thermal unit commitment via multilevel scenario trees and bundle regularization," Optim. Eng., 2019.

[13] R. Taktak and C. D'Ambrosio, "An overview on mathematical programming approaches for the deterministic unit commitment problem in hydro valleys," Energy Syst., vol. 8, no. 1, pp. 57-79, 2017.

[14] E. Finardi, E. Silva, and C. Sagastizábal, "Solving the unit commitment problem of hydropower plants via Lagrangian relaxation and sequential quadratic programming," Comput. Appl. Math., vol. 24, no. 3, pp. 317-341, 2005.

[15] C. a. Floudas and X. Lin, "Mixed integer linear programming in process scheduling: Modeling, algorithms, and applications," Ann. Oper. Res., vol. 139, no. 1, pp. 131-162, 2005.

[16] G. Alvarez, M. Marcovecchio, and P. Aguirre, "Hydrothermal Unit Commitment with Deterministic Optimization: Generation and Transmission Including Pumped Storage Units," Electron. J. Informatics Oper. Res. SADIO - Spec. Issue Dedic. to JAIIO 2017, vol. 17, no. 1, pp. 92-115, 2018.

[17] C. Cheng, J. Wang, and X. Wu, "Hydro Unit Commitment with a Head-Sensitive Reservoir and Multiple Vibration Zones Using MILP," IEEE Trans. Power Syst., vol. 31, no. 6, pp. 4842-4852, 2016.

[18] C. Cheng, C. Su, P. Wang, J. Shen, J. Lu, and X. Wu, "An MILPbased model for short-term peak shaving operation of pumpedstorage hydropower plants serving multiple power grids," Energy, vol. 163, pp. 722-733, 2018.

[19] Sala de Prensa ITAIPU, "ITAIPU BINACIONAL. La mayor hidroeléctrica del mundo en producción de energía." [Online]. Available: https://www.itaipu.gov.py. [Accessed: 30-Aug-2019].

[20] L. M. Cardenas and C. J. Franco, "Structure and Current State of the Wholesale Electricity Markets," IEEE Lat. Am. Trans., vol. 15, no. 4, pp. 669-674, 2017.

[21] V. Hinojosa, O. Ticuna, and G. Gutierrez, "Improving the Mathematical Formulation of the Unit Commitment with Transmission System Constraints," IEEE Lat. Am. Trans., vol. 14, no. 2, pp. 773-781, 2016.

[22] G. Alvarez, M. Marcovecchio, and P. Aguirre, "SecurityConstrained Unit Commitment Problem including thermal and pumped storage units: An MILP formulation by the application of linear approximations techniques," Electr. Power Syst. Res., vol. 154, pp. 67-74, 2018.

[23] A. Arce, T. Ohishi, and S. Soares, "Optimal dispatch of generating units of the Itaipú hydroelectric plant," IEEE Trans. Power Syst., vol. 17, no. 1, pp. 154-158, 2002.

[24] J. M. P. Filipe, "Optimization strategies for pump-hydro storage and wind farm coordination including wind power uncertainty," 2014.

[25] E. Lehtonen, "Production Planning of a Pumped-storage Hydropower Plant," MS-E2108 Independent Research Projects in Systems Analysis. p. 23, 2015.

[26] E. A. B. Guillén, "Estimación de la potencia eléctrica teórica disponible en Río Copinula, Jujutla, Ahuachapán," INGNOVACIÓN, vol. 4, pp. 33-50, 2012.

[27] C. Mataix Plana, Hydraulic Turbomachines: Hydraulic Turbines, Pumps, Fans. Universidad Pontificia Comillas, 2009.

[28] C. T. Cheng, X. Cheng, J. J. Shen, and X. Y. Wu, "Short-term peak shaving operation for multiple power grids with pumped storage power plants," Int. J. Electr. Power Energy Syst., vol. 67, pp. 570581, 2015.

[29] C. Liu, M. Shahidehpour, and J. Wang, "Application of augmented Lagrangian relaxation to coordinated scheduling of interdependent hydrothermal power and natural gas systems," IET Gener. Transm. Distrib., vol. 4, no. July, pp. 1314-1325, 2010.

[30] IBM Corp. and IBM, "V12. 1: User's Manual for CPLEX," Int. Bus. Mach. Corp., vol. 12, no. 1, p. 481, 2009.

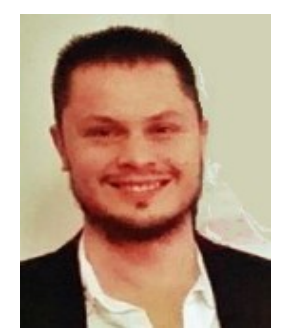

Gonzalo Exequiel Alvarez recibió el título de Ingeniero Electromecánico de la Universidad Tecnológica Nacional Facultad Regional Paraná, Argentina, en 2011. Además recibió en título de Doctorado en Ingeniería, Mención Industrial en la Universidad Tecnológica Nacional - Facultad Regional Santa Fe, en 2019. Actualmente forma parte del Consejo Nacional de Investigaciones Científicos y Técnicas (CONICET), y su lugar de trabajo es el Instituto de Desarrollo y Diseño INGAR. Sus áreas de especialización están relacionadas con la optimización de Sistemas Interconectados de energía eléctrica y de Procesos Industriales. 